\title{
Impact of Mobility on QoS in Heterogeneous Wireless Networks
}

\author{
Mikael Touati, Jean-Marc Kelif \\ Orange Labs, Issy-Les-Moulineaux, France, \\ \{mikael.touati, jeanmarc.kelif\}@ orange.com
}

\author{
Marceau Coupechoux \\ Telecom ParisTech and CNRS LTCI, Paris, France, \\ marceau.coupechoux@ telecom-paristech.fr
}

\begin{abstract}
This paper develops a model evaluating the impact of mobility in heterogeneous wireless networks by the way of analytical expressions of the spatio-temporal evolution of QoS indicators. Such formulas are obtained by introducing a multiuser averaged mobility pattern named density of users. Among the set of densities, we use a Gaussian form of this quantity, which results from a modeling method based on the maximum entropy principle. Numerical results show the temporal variations of the QoS indicators and highlight the combined effects of network heterogeneity due to the presence of macro and small cells, and traffic variations due to mobility.
\end{abstract}

Index Terms-heterogeneous networks, small cells, mobility, performance, quality of service, load and capacity analysis

\section{INTRODUCTION}

The recent and massive commercial release of wireless network connected advanced terminals and associated applications has changed users way of consuming network services. Customers did not only increase their data traffic and modify their usage, but have also increased their expectations in terms of Quality of Service (QoS) in mobility. This has led Mobile Network Operators (MNOs) to define new network planning strategies, such as the deployment of heterogeneous wireless networks. MNOs evaluate network performance by the way of QoS indicators (such as load, capacity, blocking probability), which can be derived using for example the processor sharing queue assumption with or without admission control [2]. These indicators are commonly derived in static configurations (fixed base stations and users) and temporal variations are usually introduced by the way of a user redistribution in the network following probabilistic or deterministic mobility patterns.

Authors of [4][5] analyzed the LTE wireless network heterogeneity and proposed a model evaluating the impact on coverage and throughput, showing that QoS criteria can be satisfied with reduced emitted power by the way of a spatial redeployment of stations or densification. The effect of mobility on $\alpha$-fair scheduling policies is evaluated in [3]. In [7], authors assess the problem of mobility in a micro-cell network, each cell being divided in pico-cells. User traffic demands and mobility are defined by a triplet of the form (location of arrival, size file requirement, user velocity), each component being probabilistically distributed. They obtain expressions for some characteristic times, optimal cell sizes and maximum velocities satisfying criteria leading to a successful communication. Authors of [9] model mobility in an heterogeneous network (WiFi and HSDPA cells) using migration rates following Markov
Modulated Poisson Process. Using the optimal policy of the association problem, authors show that mobility improves performance under certain assumptions.

One should also mention the huge work on mobility modeling in the literature around ad hoc networks. To have an overview of the field one can refer to the survey by Camp and al. [14] on the mobility models commonly used in simulations of ad-hoc networks. They mainly distinguish two kinds of models, the first type being entity mobility models such as random walk, random way point, Gauss-Markov or city section models and the second one being group mobility models such as exponential correlated, nomadic community or pursue mobility models. Authors of [13] evaluate the optimal placement and the optimal number of nodes in massively dense ad-hoc mobile sensor networks. This assumption leading them to describe the nodes distribution with spatial densities.

In this paper, we jointly consider mobility, network heterogeneity and user traffic, and we introduce a multiple-user mobility pattern based on a dynamic Gaussian user density. Such a quantity allows us to use a deterministic expression of the user spatial distribution with underlying probabilistic mobility patterns. We develop an analytical model leading to simple formulas of the spatio-temporal evolution of QoS indicators. By the way of numerical evaluations, we highlight the impact in time and space of both mobility and network heterogeneity on performance.

Section II introduces the system model, particularly the heterogeneous network model (II-A) in terms of its subnetworks, the mobility model (II-B) consisting in a Gaussian density based mobility pattern and the propagation model (II-C) leading to a simple Signal to Noise plus Interference Ratio (SINR) form (II-D). Section III defines the cell-type dependent indicators (loads, maximum throughputs, capacity) formulas. Section IV presents numerical results and gives an interpretation (IV-B) of the temporal evolution of the QoS indicators. Section V concludes the paper.

\section{SySTEM MOdEL}

\section{A. Network Model}

We consider a finite set $\mathcal{M}$ of omni-directional macro-cell base stations (MBSs) deployed on a hexagonal network made of several rings surrounding a central cell (see figure 1). Let $R_{c}$ be the half-distance between two neighboring MBSs, $\rho_{m}$ the MBS density and $P_{m}$ their common transmit power. In each 


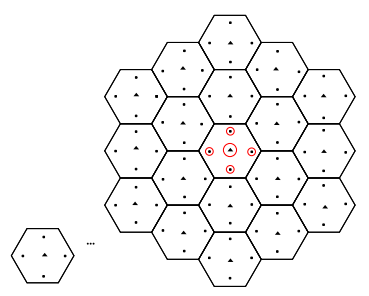

Fig. 1. Heterogeneous network obtained by the superposition of a macro-cell base stations network and a small-cell base stations network.

macro-cell, $n$ small-cell base stations (SBSs) are deployed with a regular pattern. Let $\mathcal{N}$ be the set of all SBSs of the network. Let $R_{n}$ be the average half-distance between two neighboring $\mathrm{SBSs}^{1}, \rho_{n}$ the SBS density and $P_{n}$ their common transmit power. The superposition of the MBSs and SBSs networks results in an heterogeneous network. The coverage area of each BS is defined as the set of locations, where users are served by this station. An heterogeneous cell is made of one MBS and all the SBSs deployed in its hexagon. We define the coverage area of an heterogeneous cell as the set of locations, where users are served by the MBS or any of the SBS of the concerned heterogeneous cell. We focus our analysis on the downlink.

\section{B. Mobility Model}

In any real system, the user spatial distribution at a given instant can be seen as the realization of a random point process. The temporal evolution of this distribution follows however hardly identifiable mobility patterns. The group behavior of these users may be such that, in average, the number of users per unit surface is given by a density $n(\mathbf{r}, t)$ (users $\left./ m^{2}\right)$. Such a quantity is commonly used in physics, where systems may be described at microscopic scale (atoms, molecules) or at a larger scale while considering fluid particles whose properties result from the statistical averaging of microscopic behaviors. In this paper, we focus on this larger scale and thus do not specify any individual mobility scheme, such as random way point, random-walk or Gauss-Markov model. In other words, we consider a quantity resulting from individual mobility patterns but at a larger scale than the user point process. With this approach, we can use deterministic formulas resulting from underlying probabilistic processes. Depending on the scenarios, many forms of densities may be used.

In this paper, we will concentrate on a Gaussian density of users. With this model, the user density $n(\mathbf{r}, t)$ has the following form:

$$
n(\mathbf{r}, t)=A \exp \left(-\frac{(r-\mu(t))^{2}}{2 \sigma^{2}}\right),
$$

where $\mathbf{r}=(r, \theta)$ is a position vector in polar coordinates, $A$ and $\sigma$ are constants and $\mu(t)$ is a function of time.

A mathematical argument for this choice is provided in Appendix. It uses the maximum entropy principle that allows

\footnotetext{
${ }^{1}$ In the sense of Voronoi cells.
}
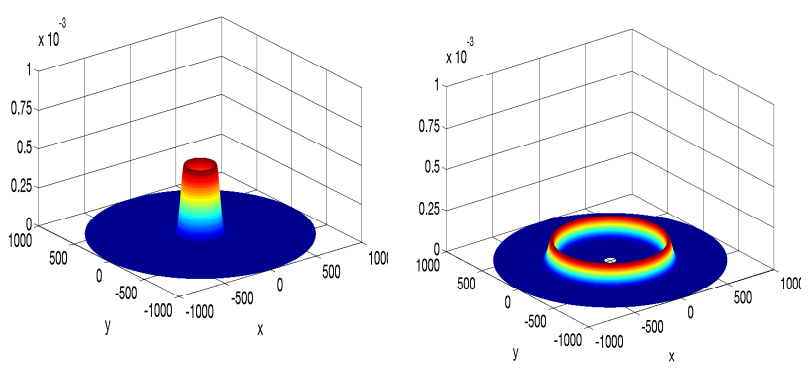

Fig. 2. Spatio-temporal diffusion of the Gaussian density of users in the plane. Left is at $\mathrm{t}=0 \mathrm{~s}$ and right at $\mathrm{t}=300 \mathrm{~s}$.

to derive the user distribution that maximizes uncertainty, when only the mean and the variance of the radial mobility pattern are known. In addition, it is interesting to focus on such a Gaussian density due to its symmetry property and the convenience it induces in terms of interpretations. Another qualitative argument in favor of this kind of density can be provided by the way of the following example. When going out of a subway entrance, users diffuse in the plane. It seems reasonable to consider in this case an average pedestrian speed of diffusion and an isotropic radial diffusion.

To give more intuition on the model, we consider here a kind of user "wave" spreading isotropically in a given area (figure 2). In such a case, the density goes through the cell at a velocity given by the temporal evolution of its mean $\mu(t)$. The spatial extension of the distribution of users is given by the parameter $\sigma$. The total amount of users in a given area is obtained by spatial integration of the density. The previously mentioned symmetric diffusion in every angular direction of the plane allows us to locate those users of the distribution who generate a non-negligible proportion of the load. Most of the users concentrate around the mean (roughly in the interval $[\mu-3 \sigma ; \mu+3 \sigma])$ and the spatial extension allows to catch the spreading trend. The slowest users locate at the back of the wave and the fastest ones locate at the front of the wave. Both cases are however less probable than the heights of the wave.

\section{Propagation Model}

We assume that the path-gain $g_{j}(u)$ between a station $j$ transmitting at power $P_{j}$ and a user $u$ only depends on the distance $r_{j}(u)$ between $j$ and $u$. The power, $P_{j, u}$, received by a mobile $u$ from $j$ can be written:

$$
P_{j, u}=P_{j} K_{j} r_{j}^{-\eta_{j}}(u)
$$

where $K_{j}$ and $\eta_{j}$ denote the path-loss propagation coefficients of station $j$. We assume constant coefficients for all stations of the same type ${ }^{2}: K_{j}=K_{m}, \eta_{j}=\eta_{m} \forall j \in \mathcal{M}$ and $K_{j}=K_{n}$, $\eta_{j}=\eta_{n} \forall j \in \mathcal{N}$.

Let define $C(\mathbf{r})$ as the peak data rate practically obtained in $\mathbf{r}$ in the absence of any other user in the cell [2]. The analytical

\footnotetext{
${ }^{2}$ For the sake of simplicity and because we focus on the mobility effect, we ignore in this paper shadowing. We will take it into account in a future work.
} 
expression of this peak rate has been obtained at Orange Labs by simulations of the LTE RF chain and consists in a simple fitting of obtained curves by a modified Shannon's formula [1] of the form:

$$
C(\mathbf{r})=a W \log _{2}(1+b \gamma(\mathbf{r}))
$$

where $a=0.6, b=1$ and $\gamma(\mathbf{r})$ is the Signal to Interference plus Noise Ratio (SINR) in $\mathbf{r}$. The dependency of the system performance on the environment and on user mobility is taken into account by their impact on the SINR, which is detailed in the next section.

\section{SINR}

We assume that every user $u$ is attached to its best server, i.e., the station providing the highest received signal power, $u$ is thus served by $j$ if and only if ${ }^{3}$ :

$$
P_{j} g_{j}(u) \geqslant P_{i} g_{i}(u) \forall i \in \mathcal{M} \cup \mathcal{N} \text {. }
$$

Let now define $k_{u}^{*} \in \mathcal{M}$ and $l_{u}^{*} \in \mathcal{N}$ the closest MBS and SBS respectively to $u$. We can rewrite the previous association rule as follows. BS $j$ is serving $u$ is given by:

$$
\begin{aligned}
& j=k^{*} \text { if } P_{k} * g_{k *}(u) \geqslant P_{l *} g_{l *}(u), \\
& j=l^{*} \text { if } P_{k *} g_{k} *(u) \leqslant P_{l *} g_{l *}(u),
\end{aligned}
$$

where we have removed the $u$ subscript in $k_{u}^{*}$ and $l_{u}^{*}$ for a reason of simplicity. Finally, we can write the worst-case SINR (all stations interfering except the serving station) as:

$$
\gamma(u)=\frac{P_{m} K_{m} r_{k *}^{-\eta_{m}} \mathbf{1}_{\left\{j=k^{*}\right\}}+P_{n} K_{n} r_{l *}^{-\eta_{n}} \mathbf{1}_{\{j=l *\}}}{I_{N}(u)+I_{M}(u)+N_{t h}}
$$

where:

$$
\begin{aligned}
I_{N}(u) & =\sum_{l \in \mathcal{N} \backslash\left\{l^{*}\right\}} K_{n} P_{n} r_{l}^{-\eta_{n}}+P_{n} K_{n} r_{l *}^{-\eta_{n}} \mathbf{1}_{\left\{j=k^{*}\right\}} \\
I_{M}(u) & =\sum_{k \in \mathcal{M} \backslash\left\{k^{*}\right\}} K_{m} P_{m} r_{k}^{-\eta_{m}}+P_{m} K_{m} r_{k^{*}}^{-\eta_{m}} \mathbf{1}_{\{j=l *\}}
\end{aligned}
$$

and $N_{t h}$ is the thermal noise power. In numerical implementations presented in section IV use the fluid model [6] to simplify the calculations of (8) and (9). We now derive in the next section the QoS indicators of the heterogeneous network.

\section{PERFORMANCES}

Let $d S(\mathbf{r})$ be an elementary surface located at $\mathbf{r}=(r, \theta)$. The total number of users in $d S(\mathbf{r})$ at $t$ is given by:

$$
n(d S(\mathbf{r}), t)=n(\mathbf{r}, t) \times d S
$$

We assume that the traffic demand is independently and identically distributed (i.i.d) among users and arrives as a Poison process of intensity $\lambda_{u}$. Flows sizes are i.i.d and given by the random variable $\sigma_{f}$. We define the traffic density as the average number of bits per unit of time and surface:

$$
\rho(\mathbf{r}, t)=n(\mathbf{r}, t) \lambda_{u} \mathbb{E}\left[\sigma_{f}\right] \quad \text { bits } / s / m^{2}
$$

\footnotetext{
${ }^{3}$ Indetermination is solved at random.
}

The traffic intensity generated by users in a surface $d S(\mathbf{r}, t)$ at $t$ is given by:

$$
d \rho_{d S}(\mathbf{r}, t)=n(d S(\mathbf{r}), t) \lambda_{u} \mathbb{E}\left[\sigma_{f}\right] \quad \text { bits } / s
$$

\section{A. Cell load}

Based on [2], we define the total load generated by a domain $D_{\mathbf{r}}=D_{\theta} \times D_{R}$ served by a unique station:

$$
\bar{\rho}_{D_{\mathbf{r}}}(t)=\int_{D_{\theta} \times D_{R}} \frac{d \rho_{d S}(\mathbf{r}, t)}{C(\mathbf{r})}=\lambda_{u} \mathbb{E}\left[\sigma_{f}\right] \int_{D_{\theta}} \int_{D_{R}} \frac{n(\mathbf{r}, t)}{C(\mathbf{r})} d \mathbf{r}
$$

Introducing (3) in (13), we obtain a general formula giving the instantaneous load of a bi-dimensional domain $D_{\mathbf{r}}$ with users density $n(\mathbf{r}, t)$ :

$$
\bar{\rho}_{D_{\mathbf{r}}}(t)=\frac{\lambda_{u} \mathbb{E}\left[\sigma_{f}\right]}{a W} \int_{D_{\theta}} \int_{D_{R}} \frac{n(\mathbf{r}, t)}{\log _{2}(1+b \gamma(\mathbf{r}))} d \mathbf{r}
$$

We now detail this load formula (14) for SBSs and MBSs.

1) Small-cell load: Let $D_{l}=D_{\theta_{l}} \times D_{R_{l}}$ be the coverage area of $\mathrm{SBS}_{l}$. In a polar coordinate system centered on $\mathrm{SBS}_{l}$, we define the angular and radial domain such that:

$$
D_{\theta_{l}}=[0 ; 2 \pi] \quad D_{R_{l}}(\theta)=\left[R_{N_{0}} ; r_{\max }(\theta)\right],
$$

where $R_{N_{0}}$ denotes the minimal distance between users and a SBS (red circles in figure 1). The extremum $r_{\max }(\theta)$ of the radial domain is the solution of (6), i.e., the maximum distance such that in the angular direction $\theta$, the power received by any user from $\mathrm{SBS}_{l}$ is greater than the power received from MBS $k^{*}$. Assuming equality of the path-loss coefficients $\left(\eta_{m}=\right.$ $\eta_{n}=\eta$ ), we obtain a simple analytical expression of $r_{\max }(\theta)$ :

$$
r_{\max , l}(\theta)=\frac{r_{k *_{l}}\left[\cos \left(\theta-\theta_{k * l}\right)-\sqrt{\cos ^{2}\left(\theta-\theta_{k * l}\right)-A}\right]}{A}
$$

with $A=\left(1-\left(\frac{P_{n} K_{n}}{P_{m} K_{m}}\right)^{-\eta / 2}\right)$ and $\left(r_{k * l}, \theta_{k * l}\right)$ the coordinates of MBS $k^{*}$ in the polar coordinate system centered on $\mathrm{SBS}_{l}$. We can thus write the load generated by users in the coverage area of $\mathrm{SBS}_{l}$ :

$$
\bar{\rho}_{l}(t)=\frac{\lambda_{u} \mathbb{E}\left[\sigma_{f}\right]}{a W} \int_{\theta=0}^{2 \pi} \int_{r=R_{N_{0}}}^{r_{\max , l}(\theta)} \frac{n(\mathbf{r}, t)}{\log _{2}(1+b \gamma(\mathbf{r}))} r d r d \theta
$$

2) Macro-cell load: We now focus on the load $\bar{\rho}_{k}(t)$ generated by the area covered by a macro-cell base station MBS $k$. To simplify its calculation, we subtract from the total load $\bar{\rho}_{k}^{m}$ generated by the hexagon cell, the load $\bar{\rho}_{l}^{m}$ associated to the area covered by every SBS of the heterogeneous cell. We thus obtain the following formula:

$$
\bar{\rho}_{k}(t)=\bar{\rho}_{k}^{m}(t)-\sum_{l=0}^{n-1} \bar{\rho}_{l}^{m}(t)
$$

The load $\bar{\rho}_{k}^{m}$ involves a numerical integration over a convex set (the hexagon), while loads $\bar{\rho}_{l}^{m}$ involve numerical integrations similar to (17), i.e., over convex sets. The serving area of a MBS is a priori non-convex. On the contrary, the right hand side of (18) involves only numerical integrations over convex areas, which makes easier the load computation. 


\section{B. Cell capacity}

Capacity may admit many definitions. We consider the one given in [2]. It is defined as the maximum average value of total traffic in bits/s (reached at the limit of overload, i.e., when load tends to 1). We have previously defined the demand of an area as the one resulting from the activity of users distributed according to a spatial density. Thus, we first define the maximum throughput per user of a MBS or a SBS as the maximum average value of individual traffic (bits/s/user) knowing that the service is uniformly distributed among all users. In case of heterogeneous cells, we define the capacity as the maximum total average traffic (bits/s), still knowing that the service is uniformly distributed among all users.

1) Small-cell maximum throughput: The maximum throughput per user served by $\mathrm{SBS}_{l}$ is given by:

$$
\begin{aligned}
Q(l, t) & =\sup _{\bar{\rho}_{l}<1} \lambda_{u} \mathbb{E}\left[\sigma_{f}\right] \\
& =a W\left(\int_{\theta=0}^{2 \pi} \int_{r=R_{N_{0}}}^{r_{\max , l}(\theta)} \frac{n(\mathbf{r}, t)}{\log _{2}(1+b \gamma(\mathbf{r}))} r d r d \theta\right)^{-1}
\end{aligned}
$$

2) Macro-cell maximum throughput: The maximum throughput per user served by MBS $k$ is given by:

$$
\begin{aligned}
Q(k, t)= & \sup _{\bar{\rho}_{k}<1} \lambda_{u} \mathbb{E}\left[\sigma_{f}\right] \\
= & a W\left(\int_{\theta=0}^{2 \pi} \int_{r=R_{M_{0}}}^{R_{e}} \frac{n(\mathbf{r}, t)}{\log _{2}(1+b \gamma(\mathbf{r}))} r d r d \theta\right. \\
& \left.\quad-\sum_{l=0}^{n-1} \int_{\theta=0}^{2 \pi} \int_{R_{N_{0}}}^{r_{\max , l}(\theta)} \frac{n(\mathbf{r}, t)}{\log _{2}(1+b \gamma(\mathbf{r}))} r d r d \theta\right)^{-1}
\end{aligned}
$$

with $R_{M_{0}}$ the minimal distance between users and any MBS and $R_{e}$ the radius of the disk equivalent in surface to the hexagonal coverage of the macro-cell.

3) Heterogeneous-cell capacity: Finally, we define the capacity of an heterogeneous macro-cell as the total number of bits per unit of time that the system is able to transmit to users it is serving. This quantity is obtained by adding all individual maximum throughput:

$$
\bar{C}_{h}(k, t)=n_{k}(t) \times Q(k, t)+\sum_{l=0}^{n-1} n_{l}(t) \times Q(l, t)
$$

where $n_{k}(t)$ denotes the total number of users served by MBS $k, n_{l}(t)$ denotes the total number of users served by SBS $l$ ( $l^{\text {th }}$ small-cell station contained in the hexagon of MBS $k$ ). Equations (17), (18), (19), (20) and (21) allow us to quickly compute the loads and capacities in a heterogeneous network.

\section{ScEnARIO AND Results}

In this section we present the numerical results obtained from the previous analytical approach. Our aim is to show the impact of mobility (due to the spatio-temporal evolution of the density of users) on QoS indicators such as loads or capacity.

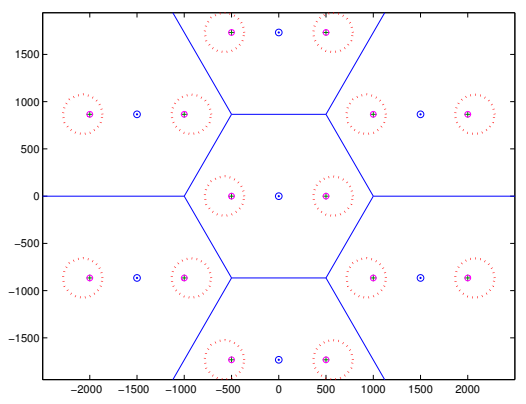

Fig. 3. Network of heterogeneous cells. Macro-stations are identified by the symbol $\odot$, small-cell stations by + . The first ring around each + gives the minimal distance to small-cells. Each small-cell coverage area (given by equation (16)) is in dotted lines.

\section{A. Assumptions}

We consider a scenario with two SBSs per heterogeneous cell. The half distance $R_{c}$ between two MBSs is $866 \mathrm{~m}$ and the distance $R_{r}$ between any SBS and the MBS at the center of its heterogeneous cell is $500 \mathrm{~m}$. The minimum distance $R_{M_{0}}$ to a MBS is $50 \mathrm{~m}$ and the minimum distance $R_{N_{0}}$ to a SBS is $30 \mathrm{~m}$. The MBS transmitting power $P_{m}$ is $43 \mathrm{dBm}$ and the SBS transmitting power $P_{n}$ is $28 \mathrm{dBm}$. The path-loss parameter $\eta$ is 3.41 . The signal bandwidth $W=10 \mathrm{MHz}$. There are $N_{t o t}=30$ users initially introduced in every macrocell and their individual average traffic intensity $\lambda_{u} \mathbb{E}\left[\sigma_{f}\right]$ is $100 \mathrm{kbits} / \mathrm{s}$.

The network layout is shown on figure 3. All hexagon cells are identical in terms of SBS deployment, we thus focus on the central cell for numerical experimentations.

We propose a Gaussian density of users with mean $\mu(t)=$ $R_{M_{0}}+3 \sigma+v t$, normalized at any instant to $N_{t o t}$. The density goes through the cell in time at velocity $v=1 \mathrm{~m} / \mathrm{s}$ (average speed of a pedestrian). Changing the average pedestrian speed $v$ would only induce changes in the time-scales of the temporal variations of the indicators shown in this section (others such as failures if considered may behave differently). The model remains valid for any other density as long as it can be written in the form $n(\mathbf{r}, t)$. If the expressions in the paper are analytically tractable then the temporal evolutions are obtained, if not they can be evaluated by numerical computations.

\section{B. Results and Interpretation}

1) Load of a small-cell: Figure 4 shows the time variations of a SBS load. These variations are due to the dispersion of users in the plane via the diffusion of their density. We particularly observe a sudden jump at time $t=230 \mathrm{~s}$, which corresponds to the first arrival of a user in the small cell coverage area. Then, we can observe the user "wave" traversing the small cell: the loads reaches a global maximum at $t=560 \mathrm{~s}$ before decreasing to zeros when the last user leaves the serving area.

Interestingly, we observe a local maximum if the load around $t=300 \mathrm{~s}$. This is due to the fact that after this time 


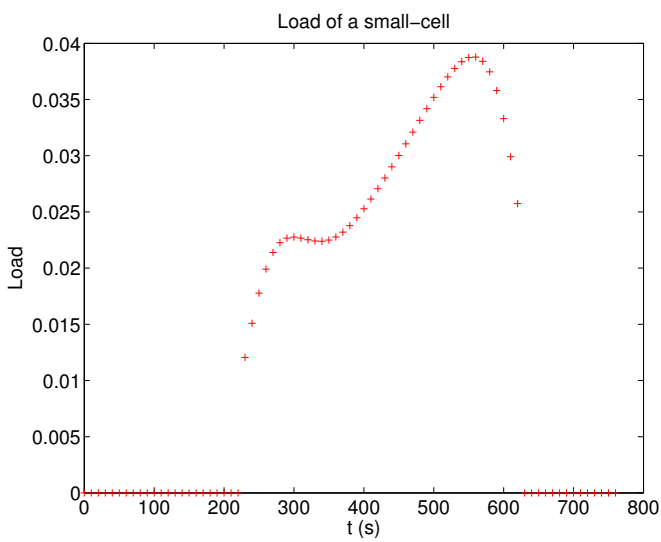

Fig. 4. Temporal evolution of the load of a small-cell.
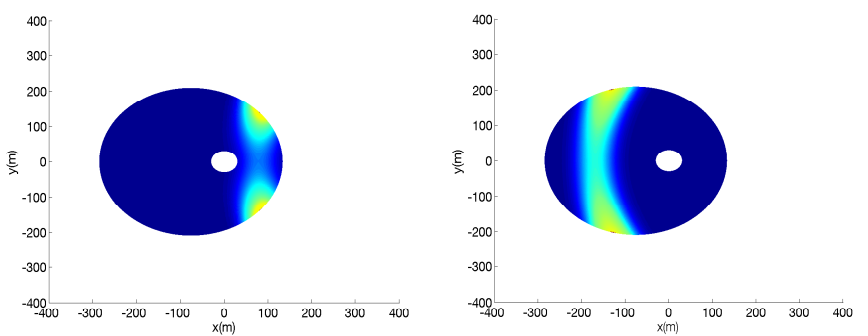

Fig. 5. Evolution of the load density in a small-cell coverage area (whithe circle is the SBS location). Left is at $\mathrm{t}=300 \mathrm{~s}$ and right at $\mathrm{t}=520 \mathrm{~s}$. Dark shades indicate low values and bright shades indicate higher ones.

instant, the peak number of users served by the SBS is reached and these users start to be in average closer to the base station. They thus tend to obtain higher physical data rates and create less load on the small cell. As the maximum of the wave starts going farther from the base station, data rates decrease and the load increases again. At $t=560 \mathrm{~s}$, this effect starts to be compensated by the fact that more and more users are leaving the serving area. Figure 5 illustrates this phenomenon by showing the SBS load density at two time instants.

2) Load of a macro-cell: Recall that users diffuse from the central MBS towards the cell edge. This means that the SINR of a user going away from its initial serving MBS decreases due to the weakening of its useful signal (path-loss effect) and the growing interferences. This explains the fact that the macro-cell load is first increasing (see figure 6). At $t=230 \mathrm{~s}$, the load is a bit stabilizing (at least increases with a lower slope). At this time instant, SBSs are indeed starting serving users, alleviating some load from the MBS. From $470 \mathrm{~s}$ to $720 \mathrm{~s}$, we observe again a high load increase. This effect being due to both users going back from small-cell to macro-cell coverage area and the global mobility towards the cell edge. At $t=720 \mathrm{~s}$, small cells do not serve any user and users start leaving the macro-cell coverage area.

3) Capacity of an heterogeneous-cell: The capacity variations observed on figure 7 result from the users weighted sum (21) of the temporal evolutions of the maximum throughputs of each serving cell (20) (19). These individual variations are

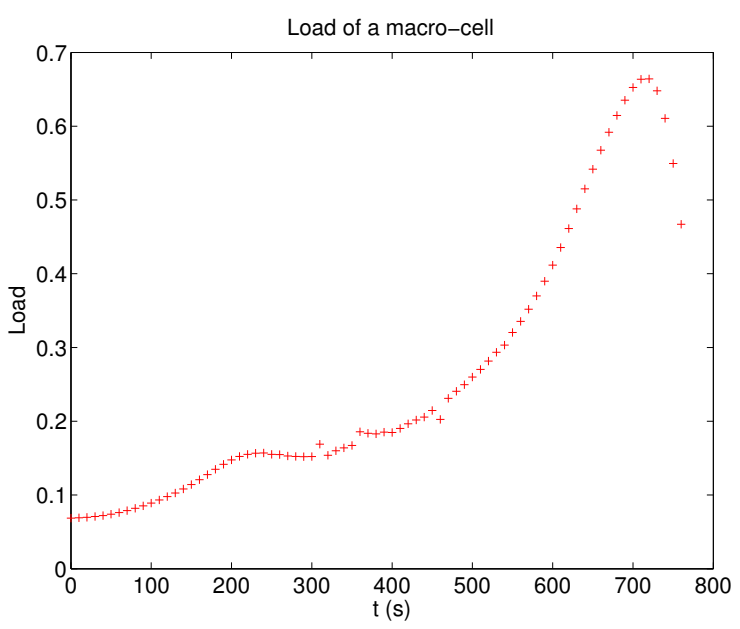

Fig. 6. Temporal evolution of the load of a macro-cell.

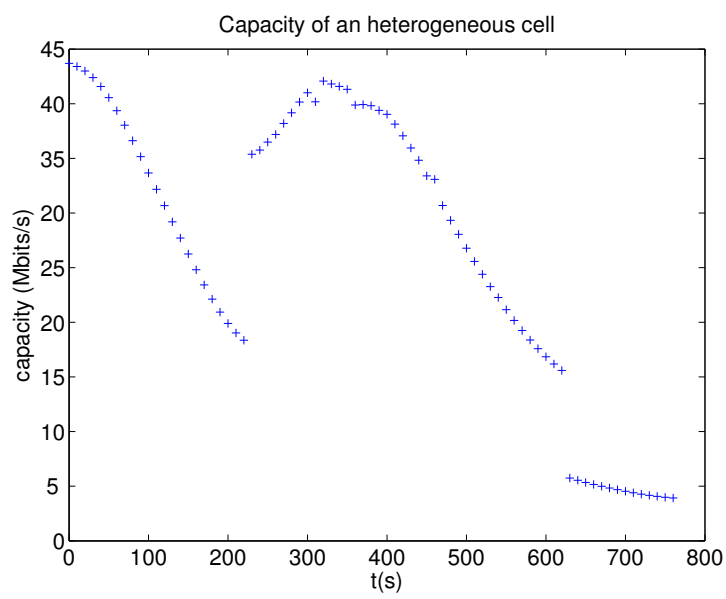

Fig. 7. Temporal evolution of the capacity of an heterogeneous cell.

obtained by inversion of the previously analyzed load variations. The gain in the heterogeneous-cell capacity obtained as a result of the introduction of small-cells is significant. When SBSs start serving $(t=230 \mathrm{~s})$, we observe an increase of $92 \%$ in capacity, rising from 18.6 Mbits/s to $35.7 \mathrm{Mbits} / \mathrm{s}$. Similarly, when the service of SBSs stops $(t=620 \mathrm{~s})$, the capacity of the heterogeneous cell decreases of $62 \%$ from $1.61 \mathrm{Mbits} / \mathrm{s}$ to $0.61 \mathrm{Mbits} / \mathrm{s}$.

\section{CONCLUSION}

In this paper, we developed an analytical model to study the impact of mobility on QoS in heterogeneous wireless networks. We introduced a multi-user averaged mobility pattern by the way of a quantity named "density of users". To illustrate this notion, we have chosen a dynamic Gaussian form. We established simple expressions of the temporal evolution of performance indicators such as load, maximum throughputs or capacity. Numerical results showed this evolution and we gave an interpretation of their variations based on the known dynamic of the Gaussian density of users. We noticed the 
stabilization of the load of a macro-cell and the significant increase of the capacity of an heterogeneous cell, both induced by the entrance of users in the coverage area of small-cells. Future work may include other forms of user densities and take into account more complex propagation models such as shadowing and fast-fading.

\section{APPENDIX}

We propose hereafter a proof justifying the Gaussian form of the density of users we use in the proposed model. This proof includes a modeling method using the maximum entropy principle; see [10], [11], [12] and references therein. Basically, we are looking for the mobility model we are legitimately able to assume when only knowing the mean and variance of the radial mobility pattern of users.

We define $\underline{\mathbf{r}}$, the matrix containing at row $i$ the two random polar coordinates $\left(r_{i}, \theta_{i}\right)$ of user $i$ among $N_{t o t}$ users and $P(\underline{\underline{\mathbf{r}}})$ the joint probability distribution of the random components of matrix $\underline{\underline{\mathbf{r}}}$. Let consider the following assumptions:

$$
\forall i, \int d \underline{\underline{\mathbf{r}}} r_{i}^{2} P(\underline{\underline{\mathbf{r}}})=\sigma_{r_{i}}^{2}=\sigma^{2} \text { and } \int d \underline{\underline{\mathbf{r}}} P(\underline{\underline{\mathbf{r}}})=1
$$

We are looking for the probability distribution of the coordinates of users maximizing uncertainty. Thus, we define the Lagrangian $L(P)$ such that:

$$
\begin{aligned}
L(P)=-\int d \underline{\underline{\mathbf{r}}} P(\underline{\underline{\mathbf{r}}}) \log (P(\underline{\underline{\mathbf{r}}})) & +\sum_{i} \gamma_{i}\left(\sigma^{2}-\int d \underline{\underline{\mathbf{r}}} r_{i}^{2} P(\underline{\underline{\mathbf{r}}})\right) \\
& +\beta\left(1-\int d \underline{\underline{\mathbf{r}}} P(\underline{\underline{\mathbf{r}}})\right)
\end{aligned}
$$

Taking the functional derivative of $L(P)$ with respect to $P$ :

$$
\frac{\delta L(P)}{\delta P}=0 \Leftrightarrow-1-\log (P(\underline{\underline{\mathbf{r}}}))-\sum_{i} \gamma_{i} r_{i}^{2}-\beta=0
$$

We obtain the following form for the distribution:

$$
P(\underline{\underline{\mathbf{r}}})=\prod_{i} P\left(r_{i}, \theta_{i}\right)
$$

with:

$$
P\left(r_{i}, \theta_{i}\right)=e^{-\left(\gamma_{i} r_{i}^{2}+\frac{\beta+1}{N_{t o t}}\right)}
$$

Using (22), we evaluate the Lagrange multipliers:

$$
e^{-(\beta+1)}=\frac{1}{\left(2 \pi \sigma^{2}\right)^{\frac{N_{t o t}}{2}} \pi^{N_{t o t}}} \text { and } \forall i, \gamma_{i}=\frac{1}{2 \sigma^{2}}
$$

Finally, we have:

$$
\forall i, \quad P\left(r_{i}, \theta_{i}\right)=\frac{1}{\sqrt{2 \pi \sigma^{2}}} \frac{1}{\pi} e^{-\frac{r_{i}^{2}}{2 \sigma^{2}}}
$$

Now assuming the knowledge of the mean of each radial component $\mathbb{E}\left[r_{i}\right]=\mu_{i}=\mu$ and its variance $\mathbb{E}\left[\left(r_{i}-\mu\right)^{2}\right]=\sigma^{2}$, we obtain (using the same method) the following spatial distribution for the coordinates of user $\mathrm{i}$ :

$$
\forall i, \quad P\left(r_{i}, \theta_{i}\right)=\frac{1}{\sqrt{2 \pi \sigma^{2}}} \frac{1}{\pi} e^{-\frac{\left(r_{i}-\mu\right)^{2}}{2 \sigma^{2}}}
$$

Let define the average number of users in an elementary surface $d S(\mathbf{r})$ (section III) as:

$$
\bar{N}(d S(\mathbf{r}))=\sum_{k} k \times P(\mathrm{k} \text { users in } d S(\mathbf{r}))
$$

This probability of having $k$ users (among $N_{t o t}$ ) in $d S(\mathbf{r})$ being given by the following binomial distribution:

$$
P(\mathrm{k} \text { users in } d S(\mathbf{r}))=\left(\begin{array}{c}
N_{t o t} \\
k
\end{array}\right) p^{k}(1-p)^{N_{t o t}-k}
$$

with $p$ the probability of a user being in $d S(\mathbf{r})$ :

$$
p=P(r, \theta) \times d S(\mathbf{r})=\frac{1}{\sqrt{2 \pi \sigma^{2}}} \frac{1}{\pi} e^{-\frac{\left(r_{i}-\mu\right)^{2}}{2 \sigma^{2}}} \times d S(\mathbf{r})
$$

Knowing the mean of a random variable following a binomial distribution, we obtain:

$$
\bar{N}(d S(\mathbf{r}))=N_{t o t} \times p=N_{t o t} \frac{1}{\sqrt{2 \pi \sigma^{2}}} \frac{1}{\pi} e^{-\frac{(r-\mu)^{2}}{2 \sigma^{2}}} \times d S(\mathbf{r})
$$

Defining the density of users as the average number of users per unit area (users $/ \mathrm{m}^{2}$ ), we finally have the Gaussian form of the expected density of section II-B:

$$
\begin{gathered}
n(\mathbf{r})=N_{t o t} \frac{1}{\sqrt{2 \pi \sigma^{2}}} \frac{1}{\pi} \exp ^{-\frac{(r-\mu)^{2}}{2 \sigma^{2}}} \\
\text { REFERENCES }
\end{gathered}
$$

\section{REFERENCES}

[1] C.E.Shannon, A mathematical theory of communication, Bell Labs Tech. J., vol.27, pp.379 - 457, 1948.

[2] T. Bonald and A.Proutiere, Wireless downlink data channels: user performance and cell dimensioning, ACM MobiCom, pp.339 - 352, Sep. 2003.

[3] S.C.Borst, N.Hegde, A.Proutiere, Mobility-driven scheduling in wireless networks, IEEE INFOCOM, pp.1260 - 1268, Apr. 2009.

[4] J-M.Kelif and W.Diego and S.Senecal, Impact of transmitting power on femto cells performance and coverage in heterogeneous wireless networks, IEEE WCNC, pp.2996 - 3001, Apr. 2012.

[5] J-M.Kelif and S.Senecal and M.Coupechoux, Impact of small cells location on performance and QoS of heterogeneous cellular networks, IEEE PIMRC, pp.2033 - 2038, Sep. 2013.

[6] J-M.Kelif and M.Coupechoux and P.Godlewski, A fluid model for performance analysis in cellular networks, EURASIP Journal on Wireless Communications and Networking, 2010.

[7] S.Ramanath and V.Kavitha and E.Altman, Spatial queuing analysis for mobility in pico cell networks, WiOpt, May 2012.

[8] P.Barsocchi, Channel models for terrestrial wireless communications: a survey, National Research Council - ISTI Institute.

[9] P.Coucheney and E.Hyon and J-M.Kelif, Mobile association problems in heterogeneous wireless networks with mobility, IEEE PIMRC, pp.3129 3133 Sept. 2013.

[10] M.Debbah, and R.R.Muller, MIMO channel modeling and the principle of maximum entropy, IEEE Transactions on Information Theory, vol.51, no.5, May 2005.

[11] M.Guillaud and M.Debbah and A.L.Moustakas, Maximum entropy MIMO wireless channel models, arXiv:cs/0612101, 2006.

[12] E.T.Jaynes,Information theory and statistical mechanics, The Physical Review, vol.106, pp.620 - 630, May 1957.

[13] A.Silva and E.Altman and M.Debbah and G.Alfano, Magnetworks: how mobility impacts the design of Mobile Networks, IEEE INFOCOM, Mar 2010.

[14] T.Camp, J.Boleng, V.Davies, A Survey of mobility models for ad hoc network research, Wireless Communications and Mobile Computing, vol.2, pp.483 - 502, 2002. 